\title{
MULTI-BASELINE INSAR ELEVATION INVERSION METHOD BASED ON THREE- DIMENSIONAL RECONSTRUCTION MODEL
}

\author{
Fenfen Hua ${ }^{\mathrm{a}, *}$, Guihui Peng ${ }^{\mathrm{a}}$, Lijun Lu ${ }^{\mathrm{b}}$, Zhaoxu Wang ${ }^{\text {a }}$ \\ ${ }^{a}$ Aerial Photogrammetry and Remote Sensing Bureau, Xi’an, Shanxi 710199, China - huafenfen_hff@163.com \\ ${ }^{\mathrm{b}}$ Chinese Academy of Surveying and Mapping, Beijing 100830, China - lulijun2002@gmail.com
}

Commission III, WG III/6

KEY WORDS: InSAR, Airborne, Multi-baseline, Three-dimensional reconstruction model

\begin{abstract}
:
The existing multi-baseline methods have some problems of low accuracy and intensive calculation. In order to solve the problems, a new multi-baseline InSAR elevation inversion method based on a rigorous geometric model instead of a simplified model is proposed in the letter. This method introduces the three-dimensional reconstruction model based on rigorous geometric model and the unknown full cycles of interferometric phase as a parameter to iteratively solve the 3-D coordinates of the target. With adopting the 3-D coordinate information of targets to connect different interferometric data, the new method obviously weakens the effects of system errors on solving the integer cycle and is more reliable than conventional multi-baseline InSAR methods. The experimental results show that the speed and accuracy of the new method are better than the existing methods.
\end{abstract}

\section{INTRODUCTION}

MBInSAR (Multi-Baseline Interferometric Synthetic Aperture Radar) technique could estimate the absolute interferometric phase or directly calculate elevation by fusing multiple interferograms, which greatly improves the performance of traditional single-baseline InSAR. And this technology gradually attracts increasing attention as it has several advantages of no need of phase unwrapping and GCPs (Ground Control Points), and reducing the accidental error effects.

Recently there are two main kinds of methods to calculate elevation by MBInSAR, one inverse elevation by absolute interferometric phase estimation, and the other directly calculates elevation.

The methods of interferometric phase estimation mainly bases on MLE (Maximum Likelihood Estimation) (Lombardini, 1996) or union pixel model (Zhang 2006, Li 2006). Both suppose that the baseline length is proportional to absolute interferometric phase which is obtained only in extremely ideal conditions. MLE method requires the numerator and denominator of the ratio of different baseline length is mutual prime number. The requirement cannot be met for all SAR system since the special multi-antenna equipment needed to be configured on SAR system. And the method based on union pixel model automatically compensates the registration error when generating interferograms. Although high quality interferograms can be generated by this method, the details will be lost in the interferograms.

Now there are a variety of methods to calculate elevation. Several methods, e.g. MLHE (Maximum Likelihood Height Estimation) (Pascazio, 2001), MRF-MAP (Markov random fields maximum a posteriori) (Ferraiuolo, 2004) and its improved algorithms (Shabou 2012, Yuan 2013), use the same probability density function to establish the relationship between the elevation and interferometric phase, and the only difference is the degree of restraining noise. However, these methods are sensitive to SAR parameter errors so that larger errors may be produced in elevation inversion even if the rigorous geometric model is introduced in these methods.

At present, there are problems of low accuracy, intensive calculation and poor application in the existing multi-baseline methods, so this paper proposes a new multi-baseline interferometric SAR elevation inversion method, named MB3DRe (Multi-baseline Three-dimensional Reconstruction). Section 2 first presents the new elevation estimation model introducing the unknown full cycles of interferometric phase as an unknown parameter to solve, then briefly describes the solution of model and the determination of initial values. Moreover, this section discusses applicability of the method. Section 3 verifies the validity of new method by using the airborne data acquired from CASMSAR (Zhang, 2012). And conclusion is given in section 4 .

\section{MULTI-BASELINE INSAR ELEVATION INVERSION}

\subsection{Three-dimensional Reconstruction Model}

Recently most methods to calculate elevation base on the simplified model, which could not meet surveying and mapping requirements. Our multi-baseline method introduces the rigorous three-dimensional construction model.

In the construction model, the 3 -D coordinates of the target $\boldsymbol{P}$ is described as the sum of the master antenna phase center $S_{1}$ and the look vector $\boldsymbol{r}$, i.e., $\boldsymbol{P}=\boldsymbol{S}_{1}+\boldsymbol{r}$. And the look vector can be written as $\boldsymbol{r}=|\boldsymbol{r}| \hat{\boldsymbol{r}}$, where $|\boldsymbol{r}|$ is the slant range and $\hat{\boldsymbol{r}}$ is the unit look vector. $S_{1}$ and $|\boldsymbol{r}|$ can be obtained from the image information. Thus the 3-D coordinates $\boldsymbol{P}$ is transformed into solving the unit look vector $\hat{\boldsymbol{r}}$. Generally speaking, $\hat{\boldsymbol{r}}$ is solved in the moving coordinate system whose original point is the

\footnotetext{
* Corresponding author
} 
master antenna phase center. The three orthogonal basis of the system are:

$$
\hat{v}=\frac{v}{|v|} \quad \hat{n}=\frac{(v \otimes b) \otimes v}{|(v \otimes b) \otimes v|} \quad \hat{w}=\frac{v \otimes b}{|v \otimes b|}
$$

Where $\boldsymbol{v}$ is the velocity vector at one moment, $\boldsymbol{b}$ is the baseline vector, $\otimes$ represents the vector cross operation. For simplicity we name the moving coordinate system as vnw system. If the transformation matrix from geocentric coordinate system to the vnw system is written as vnw $=\left[\begin{array}{lll}\hat{v} & \hat{n} & \hat{w}\end{array}\right]$, the opposite is

$$
\mathbf{v n w}_{\mathbf{i n v}}=\mathbf{v n w}^{-1}=\mathbf{v n w}^{T}=\left[\begin{array}{lll}
\hat{\boldsymbol{v}} & \hat{\boldsymbol{n}} & \hat{\boldsymbol{w}}
\end{array}\right]^{T}
$$

Since VNW is the orthogonal matrix, $\mathbf{V N W} \mathbf{W}^{-1}=\mathbf{V} \mathbf{N} \mathbf{W}^{T}$.

The unit look vector is generated from the geometric relations of InSAR, and in vnw system can be written as

$$
\hat{\boldsymbol{r}}=r_{v} \hat{\boldsymbol{v}}+r_{n} \hat{\boldsymbol{n}}+r_{w} \hat{\boldsymbol{w}}
$$

And 3-D coordinates of target can be written as:

$$
\boldsymbol{P}_{x y z}=\boldsymbol{S}_{1 x y z}+|\boldsymbol{r}| \cdot \mathbf{v n w}_{\text {inv }} \cdot\left[\begin{array}{lll}
r_{v} & r_{n} & r_{w}
\end{array}\right]^{T}
$$

Finally, we can get the elevation by transforming $\boldsymbol{P}_{x y z}$ from the geocentric coordinates to the geodetic coordinates.

\subsection{Multi-Baseline InSAR Elevation Inversion}

\subsubsection{Multi-Baseline Three-dimensional Reconstruction Model}

Different from other multi-baseline method, MB-3DRe method constitutes an iterative process in that the integer number of $2 \pi$ on a serial of interferograms is first estimated, then the coordinates of target can be calculated.

The absolute interferometric phase is expressed as $\varphi=\phi+2 k \pi$, where $\phi$ is wrapped phase generated from the interferogram, $k$ is the integer number of $2 \pi$. In single-baseline condition, phase wrapping and GCPs are absolutely essential to get the absolute phase. Instead, MB-3DRe method could avoid these processes by introducing $k$ as an unknown. Here (4) can be written as follows:

$$
\boldsymbol{F}\left(\begin{array}{llll}
P_{x} & P_{y} & P_{z} & k
\end{array}\right)=\left[\begin{array}{l}
F_{x} \\
F_{y} \\
F_{z}
\end{array}\right]=\left[\begin{array}{c}
S_{1 x}-P_{x}+|\boldsymbol{r}| \cdot\left(u_{11} \cdot r_{v}+u_{12} \cdot r_{n}+u_{13} \cdot r_{w}\right) \\
S_{1 y}-P_{y}+|\boldsymbol{r}| \cdot\left(u_{21} \cdot r_{v}+u_{22} \cdot r_{n}+u_{23} \cdot r_{w}\right) \\
S_{1 z}-P_{z}+|\boldsymbol{r}| \cdot\left(u_{31} \cdot r_{v}+u_{32} \cdot r_{n}+u_{33} \cdot r_{w}\right)
\end{array}\right]=\left[\begin{array}{l}
0 \\
0 \\
0
\end{array}\right](5)
$$

Where $\left[\begin{array}{c}P_{x} \\ P_{y} \\ P_{z}\end{array}\right]$ is the 3-D coordinates of target, $\left[\begin{array}{c}S_{1 x} \\ S_{1 y} \\ S_{1 z}\end{array}\right]$ is the 3-D coordinates of master antenna, $\left[\begin{array}{lll}u_{11} & u_{12} & u_{13} \\ u_{21} & u_{22} & u_{23} \\ u_{31} & u_{32} & u_{33}\end{array}\right]$ is the transformation matrix $\mathbf{V N W}$ inv.

As can be seen from (5), the model contains four unknowns and three equations. With one more pair of interferometric data, it would increase three equations and only one unknown. Thus when the number of interferogram exceeds two, we can get the coordinate of target without phase unwrapping and GCPs.

\subsubsection{Solution of Model}

Assuming that the number of interferograms is $n$, equations can be linearized as follows:

$$
\left\{\begin{array}{c}
\boldsymbol{F}_{10}+\frac{\partial \boldsymbol{F}_{1}}{\partial P_{x}} \mathrm{dx}+\frac{\partial \boldsymbol{F}_{1}}{\partial P_{y}} \mathrm{dy}+\frac{\partial \boldsymbol{F}_{1}}{\partial P_{z}} \mathrm{dz}+\frac{\partial \boldsymbol{F}_{1}}{\partial k_{1}} \mathrm{dk}_{1}=0 \\
\vdots \\
\boldsymbol{F}_{n 0}+\frac{\partial \boldsymbol{F}_{n}}{\partial P_{x}} \mathrm{dx}+\frac{\partial \boldsymbol{F}_{n}}{\partial P_{y}} \mathrm{dy}+\frac{\partial \boldsymbol{F}_{n}}{\partial P_{z}} \mathrm{dz}+\frac{\partial \boldsymbol{F}_{n}}{\partial k_{n}} \mathrm{dk}_{n}=0
\end{array}\right.
$$

Because $P_{x} 、 P_{y}$ and $P_{z}$ is relative to $\boldsymbol{k}$, we first solve the residuals dk, And (6) can be expressed in the matrix form as

$\boldsymbol{A} \cdot \mathbf{d k}=-\boldsymbol{L}_{A}$
Where the matrix $\boldsymbol{A}=\left[\begin{array}{ccc}\frac{\partial \boldsymbol{F}_{1}}{\partial k_{1}} & 0 & 0 \\ 0 & \ddots & 0 \\ 0 & 0 & \frac{\partial \boldsymbol{F}_{n}}{\partial k_{n}}\end{array}\right]$, the matrix $\boldsymbol{L}_{A}=\left[\begin{array}{c}\boldsymbol{F}_{10} \\ \vdots \\ \boldsymbol{F}_{n 0}\end{array}\right]$.

Using the least squares principle introduced in (7), the residuals $\mathbf{~ d k}$ can be written as:

$$
\mathbf{d k}=-\left(\boldsymbol{A}^{T} \boldsymbol{A}\right)^{-1} \boldsymbol{A}^{T} \boldsymbol{L}_{A}
$$

After getting $\boldsymbol{k}$, the coordinate of target can be generated from (4). In reality, the result with different interferometric data cannot keep the same value. So the final result can be calculated by:

$$
\boldsymbol{P}=\sum_{i=1}^{n} \boldsymbol{P}_{i} \gamma_{i} / \sum_{i=1}^{n} \gamma_{i}
$$

Where $\boldsymbol{P}_{i}$ is the result generated from the $i_{t h}$ interferometric data, $\gamma_{i}$ is the coherent coefficient of the $i_{t h}$ interferometric data. This process $(7) \sim(9)$ is repeated iteratively until the error is lower than the threshold and $\boldsymbol{k}$ does not change any more.

\subsubsection{Applicability Illustration}

The difference between different baselines is actually random. However, some methods, such as MLE and Chinese remainder theorem method, only apply to the case when the baseline length ratio is an integer. Some other methods, such as MLHE and MRF-MAP, easily get wrong elevation. But MB-3DRe method applies to most situations:

When all the baselines are approximate, that is, the differences of baseline length 、 spatial position and attitude angle are not significant, the multi-baseline model becomes single-baseline situation. Although every pair of interferometric data has not influence on each other, the unknowns, $\boldsymbol{k}$ and elevation, are determined simply by the initial elevation value and stay same in the iterative process.

When all the baseline length is very close, but the spatial position or attitude angle of baselines vary greatly from each other, the effective baselines still have obvious difference, and therefore it makes no difference to the final results.

When the baseline length ratio is an integer, it also makes no difference to the results by taking the 3-D coordinates of target into account. And when all the baseline parameters differ from each other, our method are significantly better than other methods. 
When dealing with multi-frequency data, except when the carrier frequency and baseline parameters are very close, the method could still get the fine results.

When the interferogram has a very high density, removing the flatten phase first is a good choice to solve $\boldsymbol{k}$.

\section{EXPERIMENTAL RESULTS AND ANALYSIS}

To demonstrate the performance of the above introduced algorithm, we make the experiments with the airborne X-band data acquired from CASMSAR system.

\subsection{Experiment with Airborne Data}

CASMSAR system X-band operation mode adopts the frequency of $9.6 \mathrm{GHz}$ and baseline length of $2.2 \mathrm{~m}$. The airborne data are acquired at time of 2012/10/01, 2012/10/08, and
2012/10/14 over Ruoergai, Sichuan Province of China. For the sake of simplicity, we name these data as 1001, 1008 and 1014. The specific information of data is shown in Table 1.

Due to every pair of the dual-antenna data are acquired at the same time, it could generate a high quality interferogram. However, the short wavelength and unstable attitude angles would lead to a poor interferogram generated from different pass data. Although the experimental data have the same baseline length, the special position and attitude angles are different. Therefore the data can be taken as multi-baseline data. Schematic Diagram of Airlines is shown in Figure 1:

In Figure 2, (a), (b) and (c) separately show the raw image by master antenna of 1001, 1008 and 1004, (d), (e) and (f) separately represent the interferogram generated from the dualantenna interferometric data. The left side of data is near-range position; the opposite side is far-range position.

\begin{tabular}{|c|c|c|c|}
\hline Name & $\begin{array}{l}\text { Flying height } \\
\text { (m) }\end{array}$ & $\begin{array}{c}\text { Flying } \\
\text { velocity } \\
(\mathrm{m} / \mathrm{s})\end{array}$ & $\begin{array}{c}\text { Initial } \\
\text { range } \\
(\mathrm{m})\end{array}$ \\
\hline 1001 & 6474.40 & 132.92 & 3593.67 \\
\hline 1008 & 6499.01 & 133.21 & 3654.6 \\
\hline 1014 & 6467.89 & 133.12 & 3630.60 \\
\hline & $\mid \uparrow$ & . & \\
\hline
\end{tabular}

(a) Plane Graph of Airlines (b) profiles of Airlines Figure 1. Schematic Diagram of Airlines

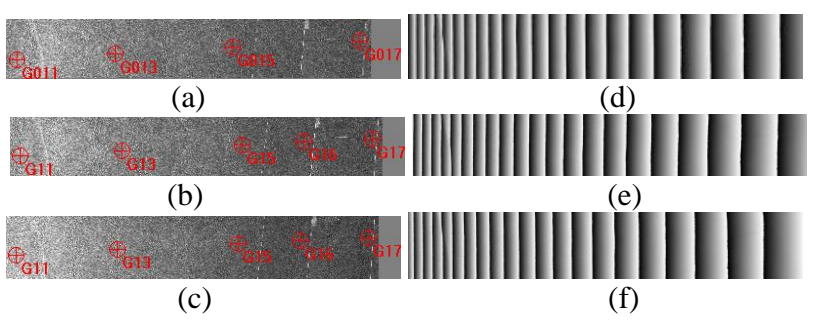

Figure 2. SAR image and interferogram of experimental area

As shown in Fig. 2, most experimental area is flat except a sharp ridge which appears as a bright line on near-range direction. And the bright lines on far-range end represent the metal fences. Corner reflectors are approximate uniformly

Table 2 Error of Airborne InSAR Height Inversion

\begin{tabular}{clrr}
\hline & Method & G11 & G13 \\
\hline Not & MLHE & -26.520 & -42.181 \\
Calibrated & MB-3DRe & 6.402 & 9.377 \\
\hline \multirow{4}{*}{ Calibrated } & MLHE & G11 & MSE of GCP \\
& MB-3DRe & -0.420 & -0.061 \\
The height maps of experimental area are shown in Figure 3: & -0.216 & 0.073 \\
& &
\end{tabular}

(a) MLHE with initial data (b) MLHE with calibrated data MLHE method.

\begin{tabular}{ccc}
$\begin{array}{c}\text { Pixel } \\
\text { size } \\
(\mathrm{m})\end{array}$ & $\begin{array}{c}\text { Data } \\
\text { size } \\
(\text { Pixel })\end{array}$ & $\begin{array}{c}\text { Doppler } \\
\text { frequency } \\
(\mathrm{Hz})\end{array}$ \\
\hline $0.29 \times 0.25$ & $1085 \times 6882$ & 372.63 \\
$0.27 \times 0.25$ & $1085 \times 6934$ & 500.87 \\
$0.25 \times 0.25$ & $1184 \times 6982$ & 408.54 \\
\hline
\end{tabular}

distributed along the range direction, separately named G11, G13, G15, G16 and G17.

In the experiment, the data are divided into two types: the initial data and the calibrated data with GCPs. The calibration method adopts the sensitivity equations to calibrate baseline length, baseline angle, initial slant range, Doppler frequency and offset of interferometric phase [The calibration method]. In calibration process, G11, G15, G17 are chosen as the control points, and G13, G16 are check points to check the accuracy of elevation. Next we use the improved MLHE (Hua, 2014) by strict geometric model and proposed method to deal with these data. In order to make two methods referring to the same geometric model, we introduce Range-Doppler model and interferometric equation into the probability density function of

As the height of test area is about $3400 \mathrm{~m}$, MLHE method searches the optimal height value in interval_[3350, 3450], and MB-3DRe method sets $3400 \mathrm{~m}$ as initial value. The precision of height inversion are shown in Table 2, Figure 3 shows the inversed height. 
Errors of initial data are mainly baseline vector, initial slant range and interferometric phase offset. As can be seen from table 2, for initial data, it exist obvious height errors, which are caused by the deviation of baseline, interferometric phase and the initial range. We can also see that MLHE and proposed method have similar errors in the G15, G16 and G17, the main reason is that the two methods adopt the rigorous geometric model, and the most errors come from system parameters. While in the G11 and G13, MLHE method has obvious errors, moreover, about 2300 columns height values in the near-range side of height map (a) is relatively low, and G11 and G13 happen to locate in this region. It illustrates that systematic errors cause the maximum value of probability density function to shift from one maxima to another one, which leads to the abrupt changes of height in the near-range area. For calibrated data, the height accuracy of two methods is similar, and height maps (b) and (d) also show the same trend.

Significantly, in Fig. 3 (a) and (b), the height maps inversed by MLHE method, there are abundant abnormal values, MB3DRe method effectively solves the problem as shown in (c) and (d). From view of execution efficiency, MB-3DRe method is $6 \sim 7$ times the MLHE as shown in table 2.

In short, MB-3DRe method has higher precision and is more efficient than MLHE.

\subsection{Analysis}

The most significant difference between MB-3DRe method and other multi-baseline elevation inversion method beside MLHE is that MB-3DRe method use three-dimensional coordinates of targets to connect different interferograms however other methods only using elevation information. Therefor MB-3DRe method has two more restrictions than others. When system parameters error or phase noise only occurs in one dimension, for instance, phase noise has great influence on elevation dimension and has little effect on planar (i.e., two dimensions of $\mathrm{X}$ and $\mathrm{Y}$ constituting a planar), MB3DRe method reduce the influence of errors on cycle number solution via decomposing in three orthogonal directions. Not only MLHE method but also other methods, except [9], use only elevation value connecting different interferograms. Although MB-3DRe method is only compared with MLHE method, still can represent most of the other methods. Therefore, MB-3DRe method is robust and has good accuracy.

When interference fringe is too dense, MLHE method usually does not work as it remains the wrapped phase value leading to the removal of flat earth effect failure. MB-3DRe method can reduce the fringe density by flat earth phase removal, so as to reduce the difficulty of solving cycle number. Therefore, in dense fringe region, MB-3DRe method does work well.

As for the implantation efficiency, in the case of GCPs, MB-3DRe method can converge in a short time. In the case of no GCPs, MB-3DRe method can run fast as well when a low precision DEM data is introduced.

\section{CONCLUSION}

The multi-baseline InSAR technique is vast importance to complex area mapping. This paper studies a multi-baseline elevation inversion method based on three-dimensional reconstruction model, which placed the cycle number as unknown parameter in equation set to be solved. Comparing with other methods, MB-3DRe method has better robustness, applicability, higher precision and speed. Because error sources under multi-baseline InSAR condition are in many aspects, the results of this paper have a small amount abnormal value. The next step will introduce the advantages of noise suppression to further improve the performance of MB-3DRe method.

\section{ACKNOWLEDGEMENTS}

Supported by The National Natural Science Foundation of China (Grant No. 41401530)

\section{REFERENCES}

Lombardini F., 1996. Absolute Phase Retrieval in a Threeelement Synthetic Aperture Radar Interferometer. In: International Conference of Radar, Beijing, China, pp. 309-312.

Zhang Qiu-ling, Wang Yan-fei., 2006. Improving the Interferometric Phase Accuracy of Distributed Satellites InSAR System with Multibaseline Data Fusion. Journal of Electronics \& Information Technology, 28(11), pp. 2011-2014.

Li Zhenfang, Bao Zheng, Li Hai, et al., 2006. Image Autocoregistration and InSAR Interferogram Estimation Using Joint Subspace Projection. IEEE Transactions on Geoscience and Remote Sensing, 44(2), pp. 288-297.

Pascazio V., Schirinzi G., 2001. Estimation of Terrain Elevation by Multifrequency Interferometric Wide Band SAR Data. IEEE Signal Processing Letters, 8, pp. 7-9.

Ferraiuolo G., Pascazio V., and Schirinzi G., 2004. Maximum a Posteriori Estimation of Height Profiles in InSAR Imaging. IEEE Trans. on GRS, 1(2), pp. 66-70.

Shabou A., and Tupin F., 2012. A Markovian approach for DEM estimation from multiple InSAR data with atmospheric contributions. IEEE Geoscience and Remote Sensing Letters, 9(4), pp. 764-768.

Yuan Zhihui, Deng Yunkai, Li Fei, et al.. 2013. Improved Multichannel InSAR Height Reconstruction Method Based on Maximum Likelihood Estimation. Journal of Electronics \& Information Technology, 35(9), pp. 2161-2167.

Zhang Jixian, Zhao Zheng, Huang, Guoman, et al. 2012. CASMSAR: An Integrated Airborne SAR Mapping System. Photogrammetric engineering \& Remote Sensing, 78(11), pp. 1110-1114.

Hua Fenfen, Zhang Jixian, Huang Guoman, et al. 2014. Improved Maximum Likelihood Height Estimation Method for Multi-Baseline InSAR Elevation Inversion. Science of Surveying and Mapping, 39(3), pp.13-18. 